\title{
Cracking of Polycrystalline Graphene on Copper Under Tension
}

Seung Ryul. $\mathrm{Na}^{1 \dagger}$, Xiaohan Wang ${ }^{2 \dagger}$, Richard D. Piner ${ }^{2}$, Rui Huang ${ }^{1}$, C. Grant Willson ${ }^{2}$, Kenneth M. Liechti ${ }^{1}$

${ }^{1}$ Department of Aerospace Engineering and Engineering Mechanics Research Center for the Mechanics of Solids, Structures and Materials The University of Texas at Austin, Austin, Texas 78712, United States

${ }^{2}$ Department of Mechanical Engineering and the Materials Science and Engineering Program The University of Texas at Austin, Austin, Texas 78712, United States

\footnotetext{
${ }^{\dagger}$ These authors (Seung Ryul Na and Xiaohan Wang) contributed equally to this work.
} 


\section{In-Situ Tensile Testing}

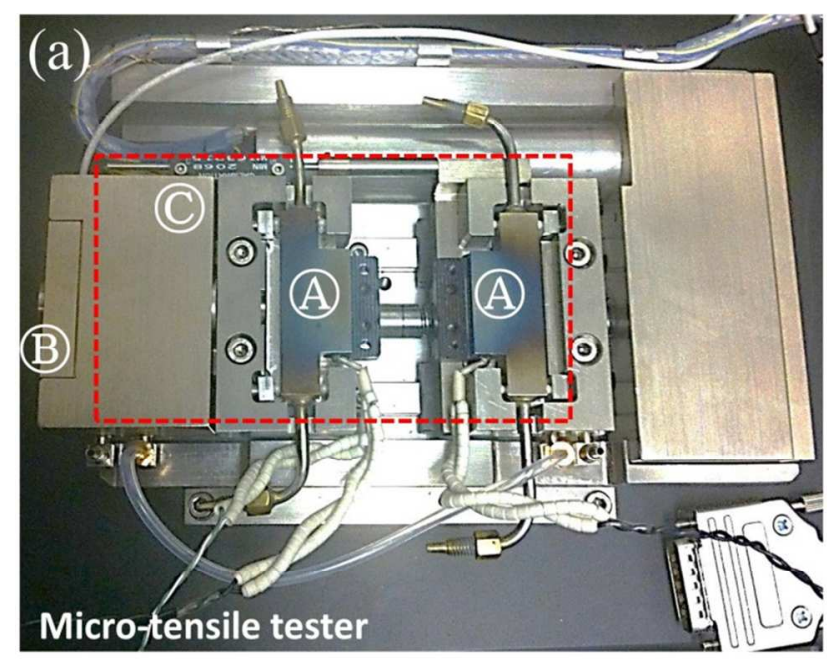

(b)

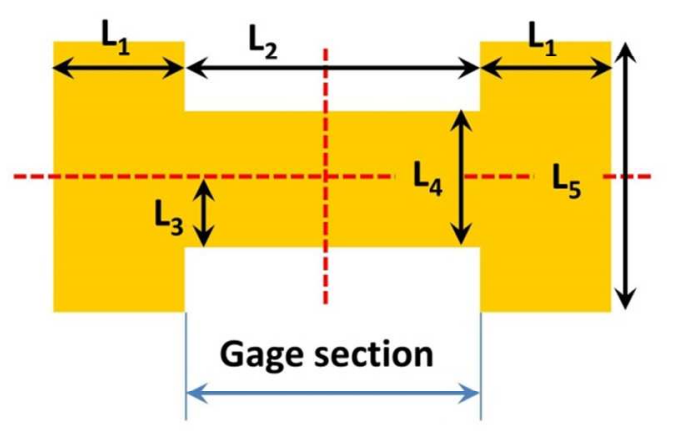

(c)
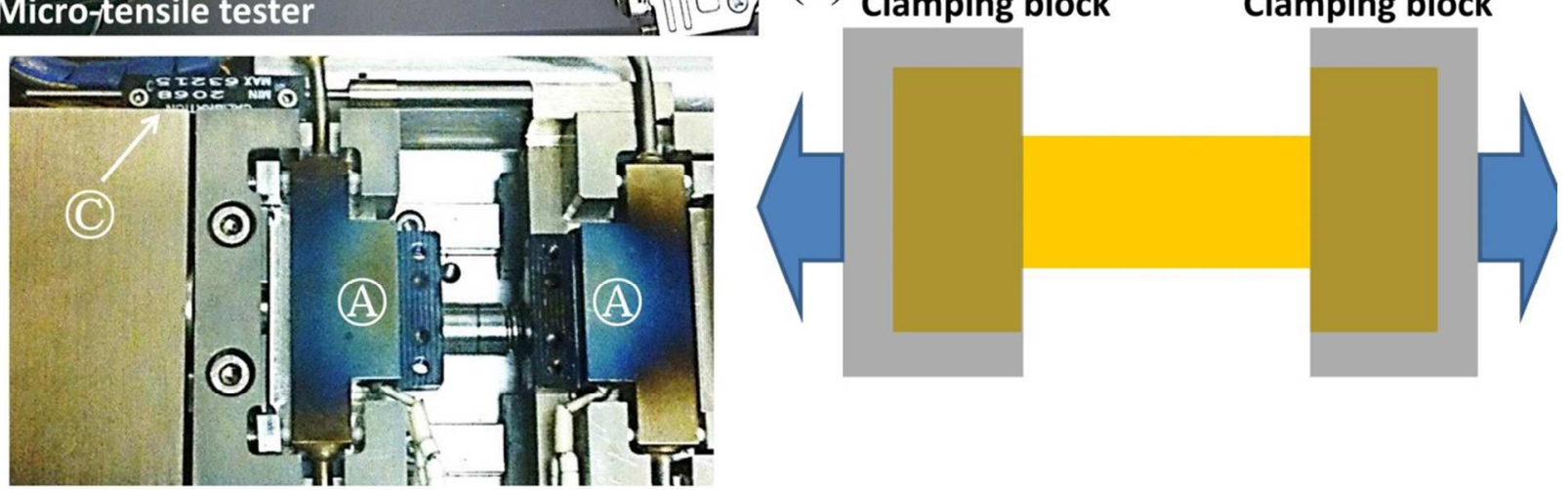

Figure S1. (a)The in-situ tensile loading device, (b) the geometry of the copper strip, and (c) schematics of the copper strips on the in-situ tensile loading device which are modeled by three series of springs.

The in-situ tensile loading device (Fig. S1a) manufactured by Deben Inc. consists of a gripping assembly (A), a load cell (B), and an LVDT (C). More details of the gripping assembly as well as the LVDT can be identified in the magnified view (below Fig. S1a) of the area inside the red box. Copper foil $(130 \mu \mathrm{m})$ was machined to the shape shown in Figure S1b with an electrical discharge machine. The dimensions of the specimens are listed in Table S1. The specimens had two gripping sections, which were aligned with the edges of the clamping blocks (Fig. S1c). After the copper was machined, the graphene was grown over its entire surface by CVD and the specimen was clamped in the testing device, so that the central portion of the gage section could be observed by SEM. 
Table S1. The geometry of the copper strips; thickness is $130 \mu \mathrm{m}$

\begin{tabular}{|c|c|}
\hline Dimension & $(\mathrm{mm})$ \\
\hline L1 & 7.62 \\
\hline L2 & 15.24 \\
\hline L3 & 5.08 \\
\hline L4 & 10.16 \\
\hline L5 & 15.24 \\
\hline
\end{tabular}

The in-situ tensile loading device was calibrated using, as a reference, the Young's modulus of the bare copper foil, which was obtained from a set of three-point bending experiments. In this configuration, the relationship between the displacement of the indenter $(\delta)$ and the reaction force $(F)$ is

$$
F=\frac{48 E I}{L^{3}} \delta
$$

where $E$ is the Young's modulus of copper, $L$ is the span between the bottom supports, $I$ is the moment of inertia associated with a rectangular section with a width $(32 \mathrm{~mm})$ and a thickness (130um). The value of the Young's modulus of copper obtained from these experiments was 105.7 $\pm 0.7 \mathrm{GPa}$, which is in agreement with reported values of about $110 \mathrm{GPa}$.

The tensile test can be modeled as two springs in series (Fig. S1c); one for the specimen gage length $\left(k_{s}\right)$ and the other for the loading device $\left(k_{m}\right)$. The overall stiffness $(k)$ was measured from the linear portions of the loading and unloading responses. The specimen stiffness was obtained from the Young's modulus of the copper foil and the specimen dimensions. Thus the machine stiffness, which was approximately $10.1 \mathrm{kN} / \mathrm{mm}$, was obtained from

$$
k_{m}=\frac{k k_{s}}{k_{s}-k} \text {. }
$$




\section{Stress-Strain Behavior}

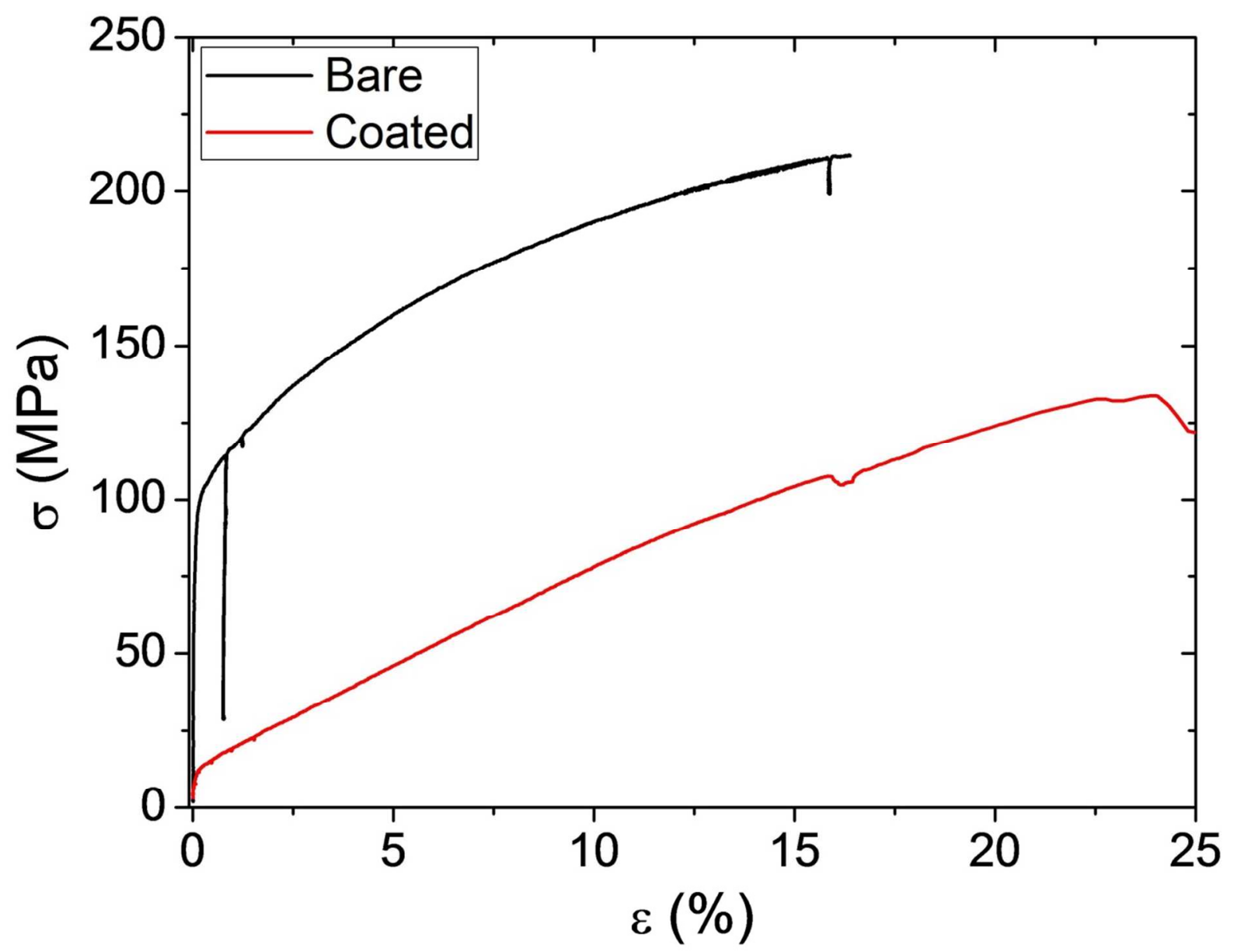

Figure S2. The stress-strain curves of bare and graphene-coated copper

The stress-strain curves of the bare and graphene-coated copper are compared in Figure S2. The Young's modulus of the bare copper foil was $107 \mathrm{GPa}$ and $101 \mathrm{GPa}$ for the coated copper, which are both comparable to the reference value of $105 \mathrm{GPa}$. The yield strength of the bare copper was approximately $110 \mathrm{MPa}$ compared to $20 \mathrm{MPa}$ for the coated sample. Such a low

yield strength is consistent with previous reports ${ }^{1}$ for annealed copper, which is what happens to the copper during CVD of graphene. The low yield strength can be explained by the classical Hall-Petch effect and the coarsening of the copper grains under annealing. 


\section{Raman Spectra}

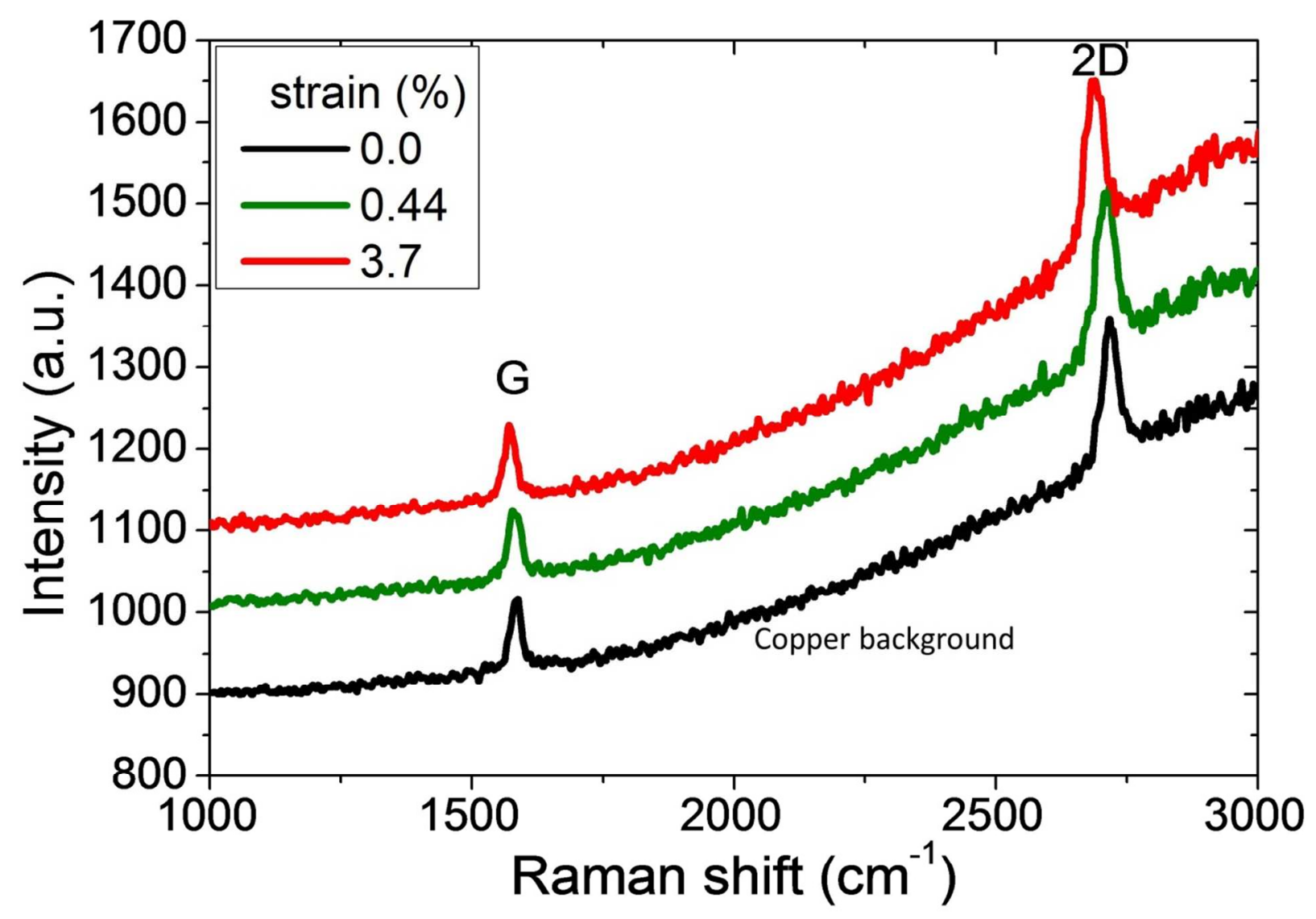

Figure S3. The Raman spectra of graphene on copper foil at three strain levels

The full Raman spectum as a function of the applied strain are shown in Figure S3. Due to the presence of the copper seed layer beneath the graphene, there was a strong background signal in addition to the $\mathrm{G}$ and $2 \mathrm{D}$ bands for graphene at approximately $1587 \mathrm{~cm}^{-1}$ and $2715 \mathrm{~cm}^{-1}$, respectively. As shown in Fig. S3, both G and 2D peaks were downshifted as the applied strain was increased. In addition, there was no D peak at $1350 \mathrm{~cm}^{-1}$, which is associated with disorder in the structure of graphene. This indicates that high-quality graphene was indeed synthesized on copper foil.

As the copper foils were deformed, a downshift and a broadening of the $G$ peak were found in the Raman spectra (Fig. S4). It is well known that the response of the G band can be 
described by the eigenvector of $\mathrm{G}^{-}$parallel to the axis of the uniaxial tension and the eigenvector of $\mathrm{G}^{+}$, which is perpendicular to the $\mathrm{G}^{-}$peak. Thus, a deconvolution of the $\mathrm{G}$ band with a Lorentzian distribution was conducted in order to separate the $\mathrm{G}^{-}$and $\mathrm{G}^{+}$peaks at various strains. Apparently, the two peaks inside the $G$ peak were becoming more and more separated with the increase in applied strain. Note that the $\mathrm{G}^{-}$peak was more sensitive to the applied strain than the $\mathrm{G}^{+}$peak.

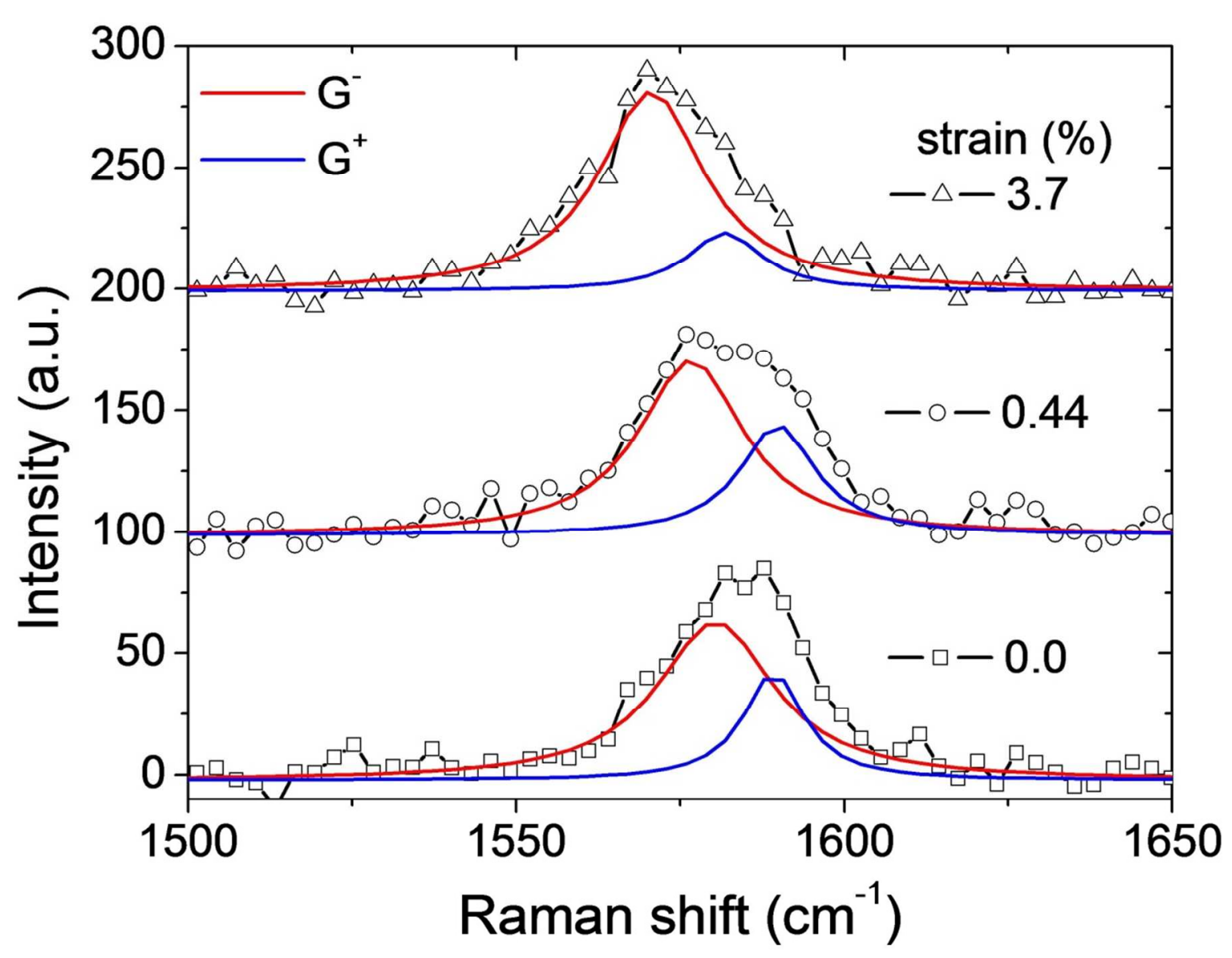

Figure S4. The Raman spectra of the $\mathrm{G}$ band at different strains 


\section{Shear Interaction Parameters}

The shear interaction between graphene and its seed copper was modeled (Fig. 5c) by an elastic stiffness $k_{s}$ followed by a constant shear strength $\tau_{c}$ associated with slip between copper and graphene. At the limit of small strain, assuming only an elastic interaction, Eq. 2 gives the energy release rate for the sequential formation of channel cracks. With a constant energy release rate equal to the fracture toughness of graphene, Eq. 2 predicts decreasing crack spacing (Fig. 5a) with increasing applied strain. However, in addition to the fracture toughness $\Gamma$ of the graphene, the interfacial stiffness $k_{s}$ is also an unknown parameter. The two parameters ( $\Gamma$ and $\left.k_{s}\right)$ were determined simultaneously from the first three data points (Table S1) by forming the quantities

$$
f_{i}=\varepsilon_{i}^{2}\left[2 \tanh \left(\lambda L_{i} / 2\right)-\tanh \left(\lambda L_{i}\right)\right]=\frac{\Gamma \lambda h}{E_{2 D}},
$$

where the subscript $i=1,2,3$ is associated with the first three data points at small applied strains. Note that the right hand side of Eq. (S1) is a constant and thus $f_{i}$ should be the same for all three data points (assuming elastic interactions only). They were evaluated for the bounds, as established in Figure 5d, of each of the measured values of crack spacing $\left(L_{i}\right)$. The six quantities are shown in Figure S5 as a function of $\lambda$. It can be seen that the only intersection, in accordance with Eq. S1, occurred between the upper bound of $f_{1}$ and the lower bound of $f_{3}$ at $\lambda=61 \mathrm{~mm}^{-1}$. The corresponding shear interaction stiffness was $1.3 \mathrm{TN} / \mathrm{m}^{3}$. The associated value of the graphene toughness was $16.5 \mathrm{~J} / \mathrm{m}^{2}$.

Table S1. The first three data points used in calculation of shear interaction parameters

\begin{tabular}{|c|c|c|}
\hline & Strain $(\%)$ & Crack spacing $(\mu \mathrm{m})$ \\
\hline 1 & 0.44 & $8.761 \pm 1.422$ \\
\hline 2 & 1.52 & $4.880 \pm 1.808$ \\
\hline 3 & 2.63 & $4.142 \pm 1.223$ \\
\hline
\end{tabular}




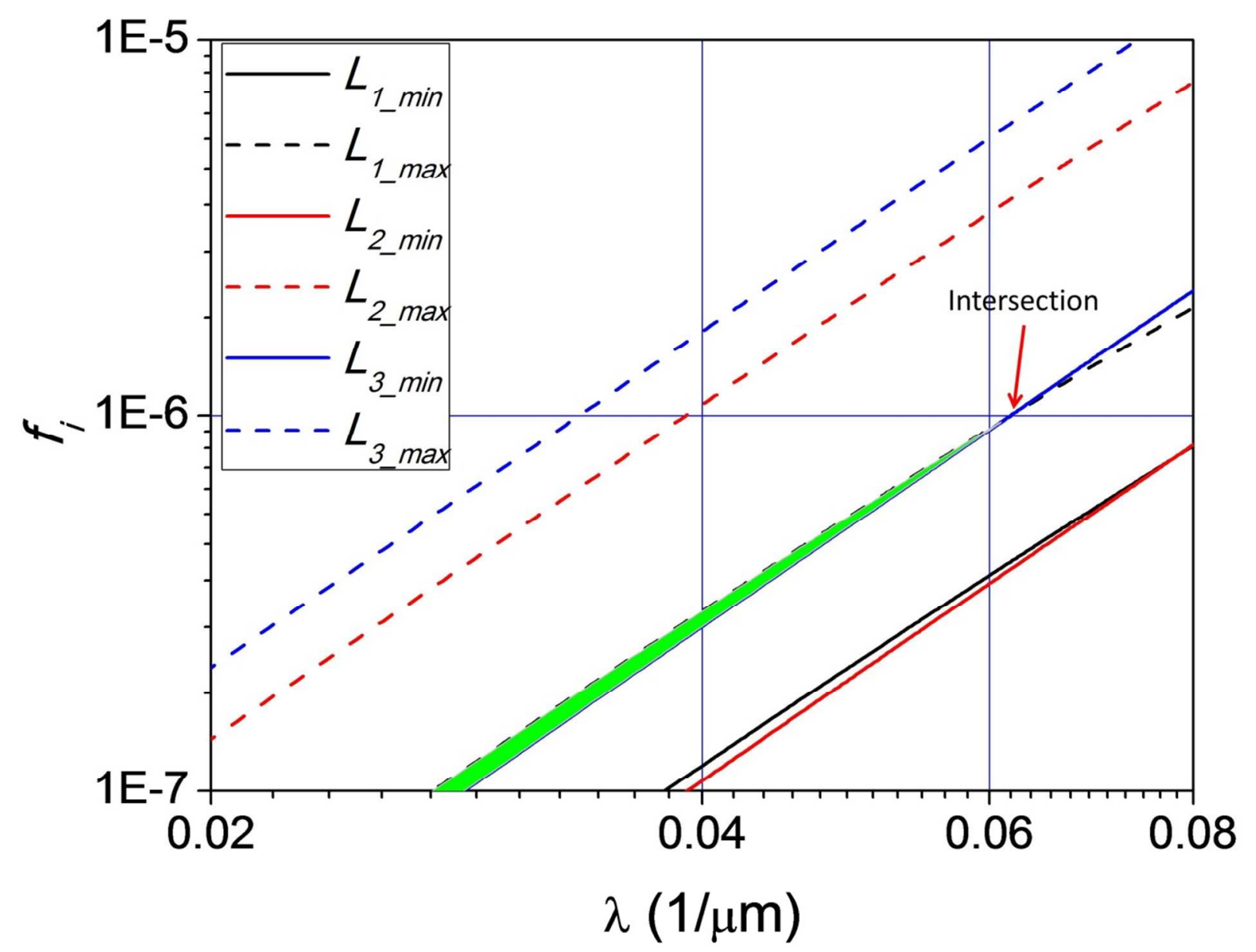

Figure S5. The domain of solutions for the crack spacing $L_{1}, L_{2}$ and $L_{3}$

Having determined the stiffness of the shear interaction and the fracture toughness of the graphene, the focus here is on the strength of the interaction $\tau_{c}$, which can be obtained from the portion of the response where the crack spacing $L$ became a minimum. Let $\tau$ be the shear stress at the interface between graphene and copper foil and $\sigma$ be the axial stress in the graphene membrane. Therefore, force equilibrium dictates that

$$
\frac{d \sigma}{d x}=\tau= \pm \tau_{c}
$$

Let $x=0$ be at the center of the graphene membrane so that $x= \pm \frac{L}{2}$ marks the edges of the graphene membrane formed by adjacent channel cracks with its associated traction free boundary condition. Thus, the normal stress distribution of the graphene at the onset of slip is given by 


$$
\sigma=-\tau_{c}(|x|-L / 2)
$$

which means that the maximum normal stress occurs at the center of the graphene membrane. The corresponding strain energy in the graphene membrane is obtained by

$$
U=\int_{-L / 2}^{L / 2} \frac{\sigma^{2}}{2 E_{2 D}} d x=\frac{\tau_{c}^{2}}{E_{2 D}} \int_{0}^{L / 2}(x-L / 2)^{2} d x=\frac{\tau_{c}^{2} L^{3}}{24 E_{2 D}} .
$$

Thus, the fracture energy of the graphene at the onset of slip (Fig. 5d) is obtained by considering the change in strain energy in the graphene in going from a spacing of $2 L$ to a spacing of $L$

$$
G=\frac{U(2 L)-2 U(L)}{h}=\frac{\tau_{c}^{2} L^{3}}{4 E_{2 D} h} .
$$

The minimum crack spacing is obtained from

$$
L_{\min }=\left(\frac{4 E_{2 D} \Gamma h}{\tau_{c}^{2}}\right)^{1 / 3}
$$

by setting $G=\Gamma$, the fracture toughness of the graphene.

\section{Reference}

1. Na, S. R.; Suk, J. W.; Tao, L.; Akinwande, D.; Ruoff, R. S.; Huang, R.; Liechti, K. M. Selective Mechanical Transfer of Graphene from Seed Copper Foil Using Rate Effects. ACS Nano 2015, 9, 1325-1335. 